\title{
ENSINO E APRENDIZAGEM DO EXAME FÍSICO: ANÁlise do PROCESSO PELO EXAME DAS PUPILAS
}

\author{
Miako Kimura* \\ Maria Sumie Koizumi**
}

Kimura, M.; Koizumi, M.S. Ensino e aprendizagem do exame físico: análise do processo pelo exame das pupilas. Rev. Esc. Enf. USP., v. 27. n. 1, p. 117-32, abr. 1993.

Este trabalho teve com objetivo analisar a aprendizagem do exame das pupilas quanto ao seu diâmetro (DP),obtido por mensuração, e ao reflexo fotomotor (RFM), avaliado mediante julgamento subjetivo. A populaçáo foi constituida por 46 alunos do $4^{2}$ semestre do Curso de Graduação em Enfermagem da USP. Os dados foram coletados em dois momentos: no laboratório de enfermagem e no campo clínico. Os resultados obtidos permitiram concluir que os alunos alcançaram altos niveis de concordância nas observaçöes feitas, além de terem adquirido uma habilidade e um conhecimento especificos. Apresentaram, porém, uma variada gama de dificuldades, sobretudo na prática em campo clínico.

UNITERMOS: Ensino-aprendizagem em enfermagem. Exame físico. Exame pupilar.

\section{INTRODUÇÃO}

O exame pupilar tem sido motivo de frequentes estudos, dada a sua importância na avaliação de pacientes com alterações neurológicas (VOIGT, 1980; LORD-FEROLI; MAGUIRE-McGINTY, 1985; WILSON et al, 1988; SOUSA, 1989).

Tais alteraçōes podem ser o denominador comum de uma variada gama de situações clínicas como trauma, infecção, neoplasia, hipoglicemia, estados epiléticos, parada cárdio-respiratória, efeito de drogas ou substâncias que levam à intoxicação exógena, efeitos anestésicos, entre outros. Apesar da aparente similaridade das disfunções cerebra is resultantes de cada uma destas causas, há importantes diferenças $\mathrm{em}$ sua fisiopatologia, podendo levar a alteraçōes, tanto no tamanho das pupilas, como na sua reatividade à luz (DUTTON, 1981; NORMAN, 1982: MARCH, 1983; TRUNKEY, 1983; ASURY, 1984; SAVOY, 1984; HALL, 1984; STEEN-HANSEN et al, 1988; ROLFSEN; DAVIS, 1989). Além disso, o exame das pupilas é um dos parâmetros mais úteis para a distinção das causas metabólicas e estruturais, podendo, inclusive, a uxiliar na localização anatômica de uma disfunção cerebral (PLUM; POSNER, 1977).

Porém, a utilização de julgamentos subjetivos para designar os diferentes padrões de reflexo fotomotor, bem como a grande variabilidade do diâmetro

* Enfermeira. Professora Doulora do Departamento de Enfermagem Médico-Cirúrgica da EEUSP.

** Enfermeira. Professora Associada do Departamento de Enfermagem Médico-Cirúrgica da EEUSP. 
pupilar podem levar a divergências nos resultados, quando um mesmo paciente é avaliado por diferentes observadorcs. Daí a preocupação de se procurar lormas de obter resultados cada vez mais objetivos nesta avaliação, a fim de que a análise da real condição do paciente e a proposição de condutas terapêuticas sejam embasadas em dados fidedignos.

Nessc sentido é que se considera importante aprimorar o ensino da habilidade para realizar o exame pupilar, entre outros aspectos do exame físico geral. Scgundo DE TORNYAY (1987), estudos na área do ensino de enfermagem têm a importante função de, não somente aprimorar o processo cducacional, mas também, em última instância, melhorar a qualidade da prática profissional.

Um aspecto importante a ser ressaltado em relação ao ensino do exame físico diz respeito às habilidades implícitas em sua realizaçāo.

A aquisição destas habilidades implica no desenvolvimento de, pelo menos, dois aspectos: o motor e o pcrceptivo (BATES; LYNAUGH, 1975). Aquelas que dependem de avaliação subjetiva do examinador, envolvem a habilidade perceptiva. É o que ocorre no caso do levantamento de dados relacionados à coloração, textura, temperatura, consistência, tamanho, forma, ruídos, odor, entre outros, cuja identificação ć intermediada pelos orgãos dos sentidos.

Para SIMÕES; TIEDMANN (1985) a aprendizagem perceptiva envolve, basicamente, quatro processos perceptivos: a detecção, a discriminação, o reconhecimento e a formação de escalas, os quais a nível comportamental, apresentam-se interrelacionados. É possível, porém, do ponto de vista teórico, distinguir cada um deles. Estes autores entendem como detecçāo a tarefa relativamente simples de determinar se um sinal ou cstímulo está presente ou não. A deteç̧ão está relacionada à determinação do limiar absoluto, ou seja, o valor mínimo de energia necessária para que um estímulo possa ser percebido. Devido à influência das diferenças individuais, a mesma intensidade de um estímulo pode não ser detectada por indivíduos diferentes.

A discriminação envolve uma situação $\mathrm{cm}$ que dois estímulos,sinais ou pistas são apresentados a uma pessoa e ela deve responder se um estímulo (o do teste) é scmelhante ou diferente de outro (o estímulo-padrão). Diz respeito à determinação do limiar diferencial, ou seja, quanto dois estímulos precisam diferir entre si para que provoquem sensações diferentes e, assim, possam ser discriminados. Na discriminação não se requer que a pessoa indique como são diferentes, mas simplesmente se há diferenças. Também neste processo, há interferência das diferenças individuais.

O reconhecimento refere-se à tarefa de identificar estímulos e saber do que se trata, além de simplesmente detectar a sua presença. Os autores citados chamam a atenção para o fato de que, no reconhecimento, o executante deve 
confiar na memória para fazer a comparação com o padrão, pois ele, na maioria das vezes, nāo está presente no momento da comparação.

No processo de aprendizagem do exame físico, é essencial que o estudante de enfermagem vá refinando a sua capacidade de perceber os sinais normais e os anormais, bem como, de distinguir as variações do normal entre diferentes pacientes.

No entanto, cmbora o labora tório de enfermagem e o campo clínico sejam utilizados para essa aprendizagem, não há estudos que demonstrem de forma controlada, a sua efetividade. Assim, julga-se que um estudo sobre fenômenos perceptivos de avaliação subjetiva e objetiva seja bastante oportuno. Outrossim, enfocando o exame das pupilas quanto aos aspectos do diâmetro pupilar (DP) e do reflexo fotomotor (RFM), poder-se-ia identificar o envolvimento da percepção visual de forma, tamanho e movimento, detectáveis pela inspeção.

Do exposto e com a metodologia de ensino comumente utilizada na enfermagem, pretende-se analisar a aprendizagem do estudante na realização do exame pupilar (DP e RFM), no que se refere:

- ao aspecto perceptivo, pela análise da concordância entre os estudantes na obtenção da medida do DP c na observação do RFM, no laboratório de enfermagem e no campo clínico;

- ao aspecto psicomotor, pela análise da execução das técnicas de medida do DP e de observação do RFM, nas duas situações de prática;

- ao conhecimento adquirido pclos estudantes sobre as técnicas em questão;

- às dificuldades sentidas pelos estudantes no aprendizado das referidas técnicas, no laboratório de enfermagem e no campo clínico.

\section{METODOLOGIA}

\subsection{População}

A população do presente estudo foi constituída de estudantes do $4^{2}$ semestre do Curso de Graduação da EEUSP, cursando a disciplina Enfermagem Médico-Cirúrgica.

Um requisito importante para a participação no estudo era que o estudante tivesse condições adequadas de acuidade visual. Foram, assim, previamente excluídos aqueles que referiram dificuldades de visão para perto, ou seja, os com hipermetropia diagnosticada, sem lentes no momento de coleta de dados.

Estabeleceu-se, também, como requisito, que o aluno não tivesse experiência anterior quanto às técnicas de exame das pupilas.

Os exames pupilares foram realizados em pacientes de ambos os sexos, internados na Unidade de Terapia Intensiva do Pronto Socorro do Hospital das Clínicas da Faculdade de Medicina da USP, pacientes estes que apresentavam alterações do nível de consciência decorrentes de distúrbios neurológicos de 
base ou conseqüentes a complicações metabólicas. Foram excluídos aqueles com Iesões oculares ou peri-orbiculares que dificultavam a realização do exame das pupilas.

\subsection{Material}

- Espátula Pupilômetro, conforme preconizado por SOUSA (1989), para mcdida do diâmetro pupilar $\mathrm{cm}$ milímetros (Anexo I).

- Lanterna para prover uma fonte luminosa artificial, utilizada na verificação da reação fotomotora das pupilas. Foram utilizadas lanternas de bolso, do mesmo tipo para todos os alunos a lim de garantir a padronização quanto à intensidade do foco obtido e ao modo de manipulação (Modelo FS-008-GUANGZHOU CHINA-Pocket flashlight).

\subsection{Métodos}

Foram reproduzidas, de forma planejada e controlada, as etapas do método de ensino composto por aula expositiva, demonstração c prática no laboratório de enfermagem e no campo clínico.

Os dados foram coletados em duas ctapas. A primeira ocorreu logo após a aula expositiva sobre o exame das pupilas, demonstração dos passos sequenciais das técnicas e prática entre estudantes no laboratório de enfermagem. Para esta prática os alunos se dividiram em pares, aleatoriamente.

A segunda etapa foi realizada no campo clínico, $\mathrm{cm}$ pacientes previamente sclecionados, obedecendo os seguintes passos:

- distribuiçāo espontânea dos pares de estudantes em um cronograma, segundo o qual 1 a 2 pares deveriam comparecer ao campo de pesquisa, cm dias e horários de sua escolha; ressalte-se que os pares foram mantidos com os mesmos membros da primeira etapa.

- seleção dos pacientes conforme critérios já definidos, feita através de consulta aos prontuários c avaliação direta do paciente pela pesquisadora;

- reforço de orientaçāo aos alunos quanto ao procedimento, atentando para a identificação de possíveis alteraçōes pupilares;

- prática dos exames pupilares em pacientes selecionados.

Foram claborados instrumentos de coleta de dados apropriados para cada ctapa. Em cada uma delas o aluno teve oportunidade de realizar o númerode exames que considerou necessário para sentir-se scguro. Esta conduta procurou levar em consideração as diferenças individuais dos estudantes no aprendizado do cxame das pupilas quanto aos seus aspectos objetivo (DP) c subjetivo (RFM). 
2.4 Critérios utilizados para a avaliação da aprendizagem das técnicas de exame das pupilas

- Concordância quanto ao exame das pupilas - tomou-se, como indicador de acerto, o nível de concordância obtido por pares de alunos na medida do DP e na obscrvação do RFM.

- Habilidade na exccução das técnicas de exame das pupilas - utilizaramsc modelos-padrōes das duas técnicas, a partir dos qua is foram elaborados "check-lists" contendo a discriminação sequencial dos ítens a screm seguidos. Os ítens foram classificados como:

* "corretos", quando exccutados conforme descrito no ítem original;

* "incorretos" ou "diferentes do padrão", quando a execução do ftem não correspondia à descriçāo do padrão ou quando havia alteração na sequência preconizada;

* "omitidos", quando o ítem não era exccutado.

- Conhecimento quanto às técnicas de exame das pupilas

- utilizou-se para esta análise, a descrição feita pelos alunos, cm impresso próprio, dos passos das duas técnicas, sendo os ítens categorizados como:

* "corretos", quando descritos de maneira idêntica ou semelhante ao modelo e desde que o significado original estivesse mantido;

* "incorretos", quando o significado cstivesse diferente do padrão;

* "omitidos", quando ausentes da descricão.

\section{RESULTADOS}

\subsection{Caracterização dos estudantes}

Do total de 52 alunos matriculados no $4^{2}$ semestre do Curso de Graduação $\mathrm{cm}$ Enfermagem da USP, foram incluídos 46 alunos que aceitaram participar das ctapas do estudo. A faixa ctária predominante foi a comprecndida entre 20 c 22 anos, incluindo-se ncla, 28 alunos $(60,9 \%)$. Do total de 46 alunos, 42 $(91,3 \%)$ cram do sexo feminino c apenas $4(8,7 \%)$, do sexo masculino.

No que diz respeito às condiçōes da acuidade visual, observou-se que a metade da classe $(50,0 \%)$ informou năo apresentar qualquer alteração da acuidade visual. Na outra metade encontravam-sc os alunos com algum tipo de deficiência visual, sendo que a maioria deles $(32,6 \%)$ fazia uso de lentes corretivas c $17,4 \%$ não as utilizava, negando, porém, qualquer dificuldade visual para lcitura próxima que justificasse a sua exclusão da população de estudo.

Nenhum dos alunos tinha experiência a nterior na avaliação do diâmetro c do reflexo pupilar, podendo-se, assim, relacionar o desempenho destes alunos ao processo ensino-aprendizagem desenvolvido. 


\subsection{Concordância quanto aos exames das pupilas}

Tabela 1. Distribuição das medidas do DP, scgundo diferença em milímetros nas duas etapas do estudo. São Paulo, 1990.

\begin{tabular}{|c|c|c|c|c|c|c|}
\hline \multirow{2}{*}{$\begin{array}{l}\text { Ftapa } \\
\text { Diferença }(\mathrm{mm})\end{array}$} & \multicolumn{2}{|c|}{$1^{a}$} & \multicolumn{2}{|c|}{$2^{a}$} & \multicolumn{2}{|c|}{ Total } \\
\hline & $n^{0}$ & $\%$ & $n^{0}$ & $\%$ & $n^{0}$ & $\%$ \\
\hline 0 & 52 & 56,5 & 44 & 47,8 & 96 & 52,2 \\
\hline 1 & 40 & 43,5 & 46 & 50,0 & 86 & 46,7 \\
\hline 2 & -- & -- & 2 & 2,2 & 2 & 1,1 \\
\hline Total & 92 & 100,0 & 92 & 100,0 & 184 & 100,0 \\
\hline
\end{tabular}

Os dados da tabela acima mostram que, do total de 184 medidas pupilares realizadas pelos alunos nas duas etapas do estudo, os valores obtidos em 96 medidas $(52,2 \%)$ foram absolutamente coincidentes. Observa-se, ainda, que 86 delas $(46,7 \%)$ tiveram diferença de $1 \mathrm{~mm}$ e apenas 2 medidas divergiram em $2 \mathrm{~mm}$.

Considerando que a diferença de $1 \mathrm{~mm}$ corresponde à margem de erro inerente ao uso da espátula pupilômetro (SOUSA, 1989), pode-se concluir que $98,9 \%$ de todas as medidas realizadas foram concordantes $(52,2 \%+46,7 \%)$.

No estudo realizado por SOUSA (1989), enfermeiros experientes obtiveram um nível de concordância de $98,3 \%$ na medida do DP. Verificou-se, portanto, que os alunos do presente cstudo apresentaram um desempenho compatível com o daqueles enfermeiros, pois na $2^{\mathrm{a}}$ etapa, a concordância entre as medidas efetuadas foi de $97,8 \%$.

Tabela 2. Distribuição dos pares de estudantes segundo concordância na medida do DP, nas duas etapas do estudo. São Paulo, 1990.

\begin{tabular}{l|c|c|c|c}
\multirow{2}{*}{ Etapa } & \multicolumn{2}{|c|}{$1^{\text {2 }}$} & \multicolumn{2}{c}{$2^{2}$} \\
\cline { 2 - 5 } & $\mathrm{n}^{\circ}$ & $\%$ & $\mathrm{n}^{\circ}$ & $\%$ \\
\hline Em ambordância & 23 & 100,0 & 22 & 95,7 \\
\hline Em um dos olhos olhos & $\cdots$ & $\cdots$ & 1 & 4,3 \\
\hline Total & 23 & 100,0 & 23 & 100,0
\end{tabular}

Tendo, ainda, como base o trabalho de SOUSA (1989) anteriormente mencionado, verifica-se na Tabela 2 que, na $1^{\text {a }}$ etapa do estudo, todos os 23 pares de estudantes obtiveram medidas concordantes em ambos os olhos. 
Na $2^{\mathrm{a}}$ etapa, apenas um dos pares não obteve esta mesma concordância pois os seus membros divergiram entre si na medida de um dos olhos.

Tabela 3. Distribuição dos pares de estudantes segundo concordância na observação do RFM, nas duas etapas do estudo. São Paulo, 1990.

\begin{tabular}{l|c|c|c|c}
\multirow{2}{*}{ Etapa } & \multicolumn{2}{|c}{$1^{2}$} & \multicolumn{2}{|c}{$2^{2}$} \\
\cline { 2 - 5 } Concordância & $\mathrm{n}^{\circ}$ & $\%$ & $\mathrm{n}^{\circ}$ & $\%$ \\
\hline Em ambos os d hos & 23 & 100,0 & 14 & 60,9 \\
\hline Em um dos ol hos & $\cdots$ & $\cdots$ & 4 & 17,4 \\
\hline Em nenhum dos olhos & -- & $\cdots$ & 5 & 21,7 \\
\hline Total & 23 & 100,0 & 23 & 100,0
\end{tabular}

Observa-se que, na $1^{\mathrm{a}}$ etapa, todos os pares de alunos foram concordantes quanto à observação do RFM. Na $2^{2}$ ctapa, apesar de $60,9 \%$ dos pares terem concordado em ambos os olhos, observa-se também que $39,1 \%$ deles discordaram em um ou em ambos os olhos.

A primeira etapa do estudo, como já foi mencionado, consistiu na observação de pupilas normais e pretendeu-se, aqui, preparar o aluno quanto aos aspectos instrumentais da técnica.

A observação de pupilas com um mesmo tipo de reação à luz, consistiu em um "julgamento de igualdade", que por si só, tem pouca influência na aprendizagem da percepção (SIMõES; TIEDMANN, 1985). Sabendo, de antemāo, que o padrão de RFM cra único é muito provável que os alunos tenham ficado compelidos a responder sempre de maneira igual, o que resultou na concordância de $100,0 \%$.

Essa $1^{\text {a }}$ etapa do processo de aprendizagem do exame pupilar teve, contudo, o importante papel de desenvolver no aluno a "imagem mental" do estímulo-padrão, ou seja, a contraçāo pupilar normal ou rápida, pcla sua observação repetida.

$\mathrm{Na} 2^{\mathrm{a}}$ etapa do estudo, diante de pacientes que poderiam apresentar diferentes tipos de RFM, o aluno deveria aprender a identificar e reconhecer novos e diferentes padrões.

Um dado importante é o de que as decisões de reconhecimento, como processos perceptivos, implicam em que o observador confie na memória para estabelecer comparaçāo entre o estímulo-padrão e o que cstá sendo observado (MAGILL, 1984). Assim, quanto mais firmemente o padrão de contração rápida (estímulo-padrão), observado na $1^{\mathrm{a}}$ etapa, tivesse ficado na memória do aluno, maior seria a possibilidade de realizar julgamentos corretos. Segundo afirmam SIMÕES; TIEDMANN (1985), o grau de dificuldade de recon- 
hecimento é determinado pelo número de alternativas, isto é, pelo número de diferentes cstímulos. Se, por exemplo, o sujeito sabe que apenas um tipo de estímulo pode ocorrer, e ele detecta algo, saberá imediatamente que se trata daquele único estímulo que pode ocorrer.

Porém, se existirem mais alternativas, as probabilidades de acertar qual o estímulo detectado, scrāo proporcionalmente menores. Foi, talvez, o que ocorreu na $2^{\mathrm{a}}$ etapa do estudo.

Tabela 4. Distribuição dos descritores do RFM, segundo concordância obtida na $2^{\mathrm{a}}$ ctapa do estudo. São Paulo, 1990.

\begin{tabular}{l|c|c|c|c|c|c}
\multirow{2}{*}{ Concordância } & \multicolumn{2}{|c|}{ Sim } & \multicolumn{2}{c|}{ Não } & \multicolumn{2}{c}{ Total } \\
\cline { 2 - 7 } Descritor & $n^{\circ}$ & $\%$ & $n^{\circ}$ & $\%$ & $n^{0}$ & $\%$ \\
\hline Rápido & 32 & 74,4 & 11 & 25,6 & 43 & 100,0 \\
\hline Lento & 8 & 38,1 & 13 & 61,9 & 21 & 100,0 \\
\hline Nāo reativo & 22 & 84,6 & 4 & 15,4 & 26 & 100,0 \\
\hline Entre rápido e lento & 2 & 100,0 & -- &.- & 2 & 100,0 \\
\hline Total & 64 & 69,6 & 28 & 30,4 & 92 & 100,0
\end{tabular}

Pelos dados da Tabela 4 pode-se verificar que os reflexos rápido e não reativo obtiveram um alto nível de observações concordantes $(74,4 \%$ e $84,6 \%$ respectivamente). Já o RFM lento foi aquele em que os estudantes demonstraram o maior nível de discordância $(61,9 \%)$.

Este resultado pode estar relacionado à dificuldade de identificar subjetivamente pequenas nuances na velocidade de contração pupilar. Os padrōes "rápido" e "não reativo" provocam sensaçōes bem definidas, o que não ocorre com o padrão intermediário "lento".

De uma forma geral, a análise do desempenho dos estudantes do ponto de vista da concordância obtida na medida do DP e na observação do RFM evidenciou resultados bastante satisfatórios.

As diferenças encontradas quanto ao desempenho na medida do DP e na observação do RFM devem ser atribuídas à própria natureza dos fenômenos examinados: de um lado, o valor de uma grandeza e de outro lado, a velocidade de contração; embora ambas sejam intermediadas pela percepçāo, quando se contou com um instrumento eficiente de medida, a possibilidade de concordância foi maior do que quando o julgamento ficou restrito a termos subjetivos. 


\subsection{Habilidade na execução das técnicas de exame das pupilas}

Tabela 5. Distribuiçāo da frequência de execução dos ítens da técnica de medida do DP, segundo as etapas do estudo. São Paulo, 1990.

\begin{tabular}{|c|c|c|c|c|c|c|c|c|c|c|c|c|}
\hline \multirow{3}{*}{$\begin{array}{l}\text { Etapa } \\
\text { Exec } \\
\text { Ítem }\end{array}$} & \multicolumn{6}{|c|}{$1^{2}$} & \multicolumn{6}{|c|}{$2^{2}$} \\
\hline & \multicolumn{2}{|c|}{ Correta } & \multicolumn{2}{|c|}{$\begin{array}{l}\text { Incorreta/ } \\
\text { dif.padrāo }\end{array}$} & \multicolumn{2}{|c|}{ Omitida } & \multicolumn{2}{|c|}{ Correta } & \multicolumn{2}{|c|}{$\begin{array}{l}\text { Incorreta/ } \\
\text { dif.padrāo }\end{array}$} & \multicolumn{2}{|c|}{ Omitida } \\
\hline & $n^{0}$ & $\%$ & $\mathrm{n}^{0}$ & $\%$ & $n^{0}$ & $\%$ & $n^{0}$ & $\%$ & $n^{0}$ & $\%$ & $n^{0}$ & $\%$ \\
\hline 1 & 9 & 19,6 & .. & -- & 37 & 80,4 & 30 & 65,2 & $-\bullet$ & - & 16 & 34,8 \\
\hline 2 & 18 & 39,1 & .. & -- & 28 & 60,9 & 46 & 100,0 & -- & -- & - & -- \\
\hline 3 & 45 & 97,8 & 1 & 2,2 & -. & -- & 45 & 97,8 & 1 & 2,2 & -- & -- \\
\hline 4 & 46 & 100,0 & -. & -- & -- & -. & 46 & 100,0 & -- & -. & -. & -. \\
\hline 5 & 36 & 78,3 & 10 & 21,7 & -. & .. & 29 & 63,0 & 17 & 37,0 & $\cdots$ & -- \\
\hline 6 & 10 & 21,7 & -. & -- & 36 & 78,3 & 46 & 100,0 & -- & .. & - & -- \\
\hline 7 & 44 & 95,7 & 2 & 4,3 & -- & -. & 45 & 97,8 & 1 & 2,2 & -- & -- \\
\hline 8 & 46 & 100,0 & -- & -. & -- & -- & 46 & 100,0 & - & -- & $\cdots$ & -- \\
\hline 9 & 36 & 78,3 & 10 & 21,7 & $\cdots$ & -- & 29 & 63,0 & 17 & 37,0 & -. & -- \\
\hline Total & 290 & 70,0 & 23 & 5,6 & 101 & 24,4 & 362 & 87,4 & 36 & 8,7 & 16 & 3,4 \\
\hline
\end{tabular}

Ítens:

1 - informar o que será feito;

2 - solicitar a fixaçāo do olhar $\mathrm{cm}$ objeto do campo visual frontal distante ou expor o globo ocular;

3 - aproximar, à $1^{\text {a }}$ pupila, a face correspondente da espátula;

4 - determinar a medida;

5 - registrar o valor obtido;

6 - solicitar que se mantenha a fixação do olhar ou expor o outro globo ocular;

7 - aproximar, à $2^{a}$ pupila, a outra face da espátula;

8 - determinar a medida;

9 - registrar o valor obtido.

A tabela 5 possibilita analisar a técnica de medida do DP quanto à exccuçāo dos seus passos pelos alunos, nas duas etapas do estudo.

Considerando-se que o modelo-padrão continha 9 ítens, o total de ítens passivos de sercm executados pelos 46 alunos era de $414 \mathrm{em}$ cada fase do estudo. Verifica-se, a partir deste dado, que o percentual de ítens corretos foi maior na $2^{\mathrm{B}}$ etapa do que na $1^{\mathrm{a}}(87,4 \%$ e $70,0 \%$, respectivamente). Ocorreram mais omissões na $1^{\mathrm{a}}(24,4 \%)$ do que na $2^{\mathrm{a}}$ etapa $(3,9 \%)$. Apesar do pequeno 
percentual, as incorreçōes e divergências do padrão foram maiores na $2^{\mathrm{a}}$ ctapa $(8,7 \%)$ do que na $1^{\circ}(5,6 \%)$.

Esta diferença quanto ao desempenho dos alunos deveu-se, principalmentc, às diferenças na frequência de exccução dos ítens relativos à abordagem inicial do paciente (ítem 1), à obtenção de condições adequadas à realização do exame (ítens 2 e 6 ) e o registro dos dados obtidos (ítens 5 e 9).

O ítem 1 "informar o que scrá feito"foi o mais omitido tanto na $1^{\circ}(80,4 \%)$ como na $2^{\mathrm{a}}$ ctapa $(34,8 \%)$ sendo, nesta fase, o único passo não cumprido.

Tabela 6. Distribuição da frequência de execução dos ítens da técnica de observação do RFM, segundo as etapas do estudo. São Paulo, 1990.

\begin{tabular}{|c|c|c|c|c|c|c|c|c|c|c|c|c|}
\hline \multirow{3}{*}{$\begin{array}{l}\text { Etapa } \\
\text { Excc } \\
\text { Item }\end{array}$} & \multicolumn{6}{|c|}{$1^{2}$} & \multicolumn{6}{|c|}{$2^{a}$} \\
\hline & \multicolumn{2}{|c|}{ Correta } & \multicolumn{2}{|c|}{ Dif.padrāo } & \multicolumn{2}{|c|}{ Omitida } & \multicolumn{2}{|c|}{ Correta } & \multicolumn{2}{|c|}{ Dif.Padrāo } & \multicolumn{2}{|c|}{ Omitida } \\
\hline & $n^{\circ}$ & $\%$ & $\mathrm{n}^{\mathrm{o}}$ & $\%$ & $\mathrm{n}^{\circ}$ & $\%$ & $\mathrm{n}^{0}$ & $\%$ & $\mathrm{n}^{\circ}$ & $\%$ & $n^{0}$ & $\%$ \\
\hline 1 & 5 & 10,9 & -- & -- & 41 & 89,1 & 21 & 45,7 & -- & -- & 25 & 54,3 \\
\hline 2 & 12 & 26,1 & -- & -- & 34 & 73,9 & 46 & 100,0 & -- & -- & -- & -. \\
\hline 3 & 46 & 100,0 & -- & -- & -- & -- & 46 & 100,0 & -. & -- & -- & -- \\
\hline 4 & 31 & 67,4 & -- & -- & 15 & 32,6 & 44 & 95,7 & -- & -- & 2 & 4,3 \\
\hline 5 & 28 & 60,9 & 18 & 39,1 & -- &.- & 25 & 54,3 & 21 & 45,7 & $-\cdot$ & -- \\
\hline 6 & 8 & 17,4 & -. & -- & 38 & 82,6 & 45 & 97,8 & -- & -- & 1 & 2,5 \\
\hline 7 & 46 & 100,0 & -- & - & $\cdots$ & -. & 46 & 100,0 & -- & -- & -. & -- \\
\hline 8 & 27 & 58,7 & -. & $\cdots$ & 19 & 41,3 & 43 & 93,5 & -- & -- & 3 & 6,5 \\
\hline 9 & 28 & 60,9 & 18 & 39,1 & -. & -- & 25 & 54,3 & 21 & 45,7 & -- & -- \\
\hline Total & 231 & 55,8 & 36 & 8,7 & 147 & 35,5 & 341 & 82,4 & 42 & 10,1 & 31 & 7,5 \\
\hline
\end{tabular}

Ítens

1 - informar o que será fcito;

2 - solicitar a fixação do olhar em objeto do campo visual frontal distante ou expor o globo ocular;

3 - incidir o foco de luz na $1^{2}$ pupila, observando a reação pupilar;

4 - retirar o foco de luz e voltar a incidí-lo;

5 - registrar o descritor observado;

6 - solicitar que se mantenha a fixação do olhar; ou expor o outro globo ocular;

7 - incidir o foco de luz na $2^{\mathrm{a}}$ pupila;

8 - retirar o foco de luz e voltar a incidí-lo;

9 - registrar o descritor observado. 
Demonstra-se, na Tabela 6, o comportamento dos alunos na execução da técnica de observação do RFM, quanto aos seus acertos, divergências e omissōes aos passos do padrão preconizado, nas duas etapas do estudo.

Observa-se, como na técnica anterior, que o percentual de ítens executados corretamente foi maior na $2^{\mathrm{a}}$ etapa $(82,4 \%)$ do que na $1^{\mathrm{a}}(55,8 \%)$; as omissões e divergências quanto aos ítens do padrāo foram relativamente pequenas na $2^{3}$ fase do cstudo, $(10,1 \%+7,5 \%=17,6 \%)$. Já na primeira fase estes comportamentos, alcançaram um percentual bastante elevado $(44,2 \%)$, sobretudo pela omissão de ítens $(35,5 \%)$.

Se na etapa desenvolvida no laboratório de enfermagem alguns ítens foram pouco valorizados, e, portanto, omitidos, no contexto de prática em campo o mesmo não ocorreu.

Ressalte-se, porém, que também na observação do RFM o ítem relativo à abordagem inicial do paciente foi o mais omitido dentre todos. Mesmo estando diante de pacientes reais, mais da metade dos alunos (54,3\%) avaliaram o RFM sem informá-los sobre o que seria feito, assim como $34,8 \%$ deles haviam medido o DP da mesma forma (Tabela 5).

Os ítens relativos ao registro dos dados foram aqueles que os alunos executaram de maneira diferente do padrão.

Portanto, foi na segunda etapa que o desempenho dos alunos mais se aproximou do padrão inicial, com mais de $80,0 \%$ dos ítens cumpridos corretamente, tanto em uma como em outra técnica. 


\subsection{Conhecimento quanto às técnicas de exame das pupilas}

Tabela 7. Distribiução da descrição dos ítens das técnicas de medida do DP e de observação do RFM. São Paulo, 1990.

\begin{tabular}{|c|c|c|c|c|c|c|c|c|}
\hline \multirow{3}{*}{$\begin{array}{c}\text { Técnica } \\
\text { Descriçāo } \\
\text { item }\end{array}$} & \multicolumn{4}{|c|}{ DP } & \multicolumn{4}{|c|}{ RFM } \\
\hline & \multicolumn{2}{|c|}{ Correta } & \multicolumn{2}{|c|}{ Omitida } & \multicolumn{2}{|c|}{ Correta } & \multicolumn{2}{|c|}{ Omitida } \\
\hline & $n^{0}$ & $\%$ & $\pi^{\circ}$ & $\%$ & $n^{\circ}$ & $\%$ & $\mathrm{n}^{\circ}$ & $\%$ \\
\hline 1 & 42 & 91,3 & 4 & 8,7 & 36 & 78,3 & 10 & 21,7 \\
\hline 2 & 42 & 91,3 & 4 & 8,7 & 41 & 89,2 & 5 & 10,8 \\
\hline 3 & 40 & 87,0 & 6 & 13,0 & 45 & 97,8 & 1 & 2,2 \\
\hline 4 & 40 & 87,0 & 6 & 13,0 & 26 & 56,5 & 20 & 43,5 \\
\hline 5 & 43 & 93,5 & 3 & 6,5 & 43 & 93,5 & 3 & 6,5 \\
\hline 6 & 35 & 76,1 & 11 & 23,9 & 40 & 87,0 & 6 & 13,0 \\
\hline 7 & 40 & 87,0 & 6 & 13,0 & 45 & 97,8 & 1 & 2,2 \\
\hline 8 & 40 & 87,0 & 6 & 13,0 & 26 & 56,5 & 20 & 43,5 \\
\hline 9 & 43 & 93,5 & 3 & 6,5 & 43 & 93,5 & 3 & 6,5 \\
\hline Total & 365 & 82,2 & 49 & 11,8 & 345 & 83,3 & 69 & 16,7 \\
\hline
\end{tabular}

Para se aferir mais acuradamente o resultado da aprendizagem das técnicas de exame do DP e do RFM solicitou-se que o aluno descrevesse, livremente, os seus passos, de modo a fazer emergir do próprio aluno, o que efetivamente ficou retido ao final do processo.

A tabela 7 demonstra a frequência com que cada uma das duas técnicas foram descritas pelos alunos, após o treinamento em campo.

Comparado ao padrão, verifica-sc que o total de ítens descritos corretamente foi bastantc elevado - 82,2\% para o DP e $83,3 \%$ para o RFM.

A omissão em percentuais elevados restringiu-se a alguns ítens. Não houve descriçāo de ítens com significado diferente do padrāo, ou seja, incorretos.

O ítem 1 "informar o que será feito" foi lembrado na descrição da técnica de medida do DP por $91,3 \%$ dos alunos e por $78,3 \%$ na de observação do RFM. Os ítens 5 e 9, referentes ao registro de dados, foram lembrados na descrição por $\mathbf{9 3 , 5 \%}$ dos alunos, apesar de não terem sido citados na sequência preconizada, tanto na primeira técnica como na segunda.

De forma geral, pode-se considerar que o conhecimento reproduzido quanto às técnicas esteve bastante próximo daquilo que foi ensinado. 
Este resultado deve-se, talvez, ao fato de que a descrição foi feita imediatamente após a conclusão da $2^{a}$ etapa, o que facilitou a rememoração dos passos.

Também porque os alunos estavam ainda numa fase inicial de aprendizagem em que o refinamento da informação e o estabelecimento de canais preferenciais que leveriam a uma síntese da informação, ainda não haviam se precessado integralmente, apesar de já detectar-se algum grau de modificaçāo.

Apesar dos indícios de que a aprendizagem tivesse ocorrido, o que se observou pela aquisição de habilidade e de um conhecimento específico, os alunos apresentaram diferentes tipos de dificuldades na realização do exame das pupilas.

\subsection{Dificuldades sentidas pelo estudante}

Da mesma forma como ocorreu na execução das técnicas, a menção de dificuldades vinculou-se diretamente ao contexto em que as práticas foram desenvolvidas. Como era de se esperar, as dificuldades foram muito mais sentidas na prática em campo do que na situação de laboratório.

Nesta primeira fase do cstudo 15 alunos $(32,6 \%)$ mencionaram dificuldades na avaliação do DP e apenas $4(8,7 \%)$, na avaliaçāo do RFM. É perfeitamente compreensível que, nesta etapa, os alunos praticamente não tivessem problemas em observar o RFM, pois tratavam-se de pupilas normais, com um padrão similar de reaçāo fotomotora.

Quanto ao exame do DP, a maioria das dificuldades $(64,7 \%)$ referiu-se a condiçōes de visualização da pupila, tais como a cor escura da íris e a variação do DP durante o exame, bem como à iluminação ambiental $(29,4 \%)$. Dificuldades relacionadas à utilização do instrumento de medida foram mencionadas apenas três vezes (uma na $1^{\mathrm{a}}$ e tapa e duas na $2^{2}$ ). Como no estudo de SOUSA (1989), comprova-se aqui também a facilidade com que se aprende a usá-la, tendo em vista que os alunos deste estudo a utilizaram pela primeira vez.

O problema de distinguir a pupila da íris de cor escura está relacionado à percepção de forma, na qual está envolvida a questão da figura e fundo. A figura é a parte do campo perceptual que aparece mais estruturada, com uma forma distinta e é o que a pessoa reconhece como objeto de sua atenção. Como fundo, entende-se aquela parte menos diferenciada contra a qual a figura é contrastada (TRAVERS, 1977). Para que ocorra a percepção da figura (pupila), é necessário que o contorno seja nítido em relação ao fundo (íris); na situação referida pelos alunos, algumas vezes, tal contraste nāo foi observado, decorrendo daí, a maior dificuldade em medir a pupila. Lembrando, também, que a superfície do globo ocular é brilhante a iluminação do ambiente, ao provocar uma reflexão da luz, pode ter dificultado ainda mais a visualizaçăo da pupila. 
Um cuidado especial deve ser tomado, portanto, na escolha do ângulo de focalização da luz a fim de minimizar o problema.

A análise conjunta dos tipos de dificuldades apontadas evidencia que elas foram consequentes às condições do paciente, do próprio cstudante e do ambiente, bem como à presença de diferentes padrōes de RFM a serem discriminados.

Com relaçāo às condiçōes do paciente, além da íris escura, foram mencionados também a presença de ferimentos na face, a posição do globo ocular, os movimentos oculares e a falta de colaboração do paciente a ser examinado.

A indicação de que o ambiente havia contribuído para dificultar as avaliaçōes foi decorrente das codiçōes de iluminaçāo e da presença de aparethos ao redor do paciente. Algumas vezes, dependendo da disposição do leito do paciente na UTI foi necessário redirecionar o foco de luz a fim de não prejudicar a visualização das pupilas, porém, mantendo-se a iluminação constante para um mesmo par de alunos observadores.

A presença de aparelhos é inerente ao tratamento intensivo e, dependendo da quantidade deles, há dificuldade, até mesmo, de acesso ao paciente. Em se tratando de alunos ainda inexperientes com esse tipo de situação, acredita-se que tenha se constituído em uma dificuldade aínda maior.

De fato, essa condição dos estudantes foi motivo de conflito, manifestando, eles próprios, o medo de chegar perto do paciente, de machucá-lo, a sensaçāo de estar perturbando-o, a dificuldade de abordar um paciente inconsciente, o receio de desligar fios a ele conectados, a falta de destreza manual.

Toda essa gama de sentimentos e dificuldades demonstrada pelos alunos deste trabalho atestam a assertiva de KOIZUMI (1976) de que cuidar de pacientes em UTI é sempre uma situação de tensão. A abordagem de pacientes em estado grave é uma dificuldade encontrada mesmo em alunos de estágios mais avançados, como constataram essas autoras, em alunos de Habilitação em Enfermagem Médico-Cirúrgica.

Porém, como fazer para minimizar os efeitos dessa situação e fazer com que os estudantes possam vivenciar essa prática de forma menos traumatizante e, prestar uma boa assistência de enfermagem?

A experiência das autoras citadas é de que esses objetivos podem ser alcançados pela utilização de estratégias que facilitem a aproximação do aluno ao paciente. Acreditam que a realização do exame físico é um meio adequado para propiciar essa aproximação, pois ele possibilita ao aluno mobilizar seu foco de atenção, do medo de não saber como atuar, para a realização de uma atividade com objetivos pré-determinados.

Auxiliar os alunos a superar dificuldades de abordagem e de relacionamento com pacientes não é tarefa fácil e as mudanças nem sempre ocorrerão sem conflitos. Porém, tendo como premissa o valor do aspecto afetivo na 
assistência de enfermagem, aliado aos instrumentais, todo esforço deverá ser empenhado nesse sentido.

Fica evidente, em todo esse processo de ensino e aprendizagem, a grande responsabilidade do professor na formação dos alunos, não somente na instrumentalizaçāo técnico-científica, mas no seu desenvolvimento integral.

\section{CONCLUSÕES}

- Em relação à habilidade perceptiva, a concordância entre os alunos foi maior no laboratório de enfermagem do que no campo clínico; foi maior a concordância na medida DP do que na observação do RFM e, neste último, a discordância mais acentuada ocorreu no padrăo intermediário.

- Quanto à habilidade em executar as técnicas houve melhor desempenho dos alunos no campo clínico do que no laboratório; o ítem ${ }^{1}$, referente à informação sobre que seria feito, foi o mais omitido pelos alunos na execução das duas técnicas, tanto no laboratório como no campo clínico.

- O conhecimento incorporado pelos alunos foi bastante próximo àquele que foi originalmente apresentado e as diferenças foram relacionadas, principalmente, à abordagem do paciente e ao registro dos dados obtidos.

- Os alunos participantes deste estudo, apesar de terem adquirido habilidade e conhecimento específicos, apresentaram variados tipos de dificuldades na realização do exame das pupilas, sobretudo nos pacientes.

- De forma geral, as dificuldades mais sentidas foram aquelas relacionadas às condiçōes do paciente, do próprio estudante e do ambiente, assim como à discriminação dos diferentes padrões de RFM.

KIMURA, M.; KOIZUML, M.S. The teaching-leaming process of physical examination: it's analysis by the pupillary exam. Rev. Esc. Enf. USP., v. 27, n. 1, p. 117-32, abr. 1993.

The goal of this study was to analyse the teaching-learning process of the pupil examination, in two aspects: the pupil diameter (DP), obtained by measurement and photomotor reflex (RFM), by subjective judgement. The sample was composed by 46 students of 4 th semester in Nursing Undergraduate Course of University of São Paulo. The study was developed in two phases: the first was at nursing laboratory and the second, at clinical practice. The findings seemed to indicate that the learning was done because there was high level of agreement among students in their observation, development of an ability and a specific knowledge. The major difficulties was at clinical practice.

UNITERMS: Nursing teaching-leaming process. Physical examination. Pupillary exam. 


\section{REFERENCIAS BIBLIOGRÁFICAS}

ASBURY, A.J.; LEAR, GA.; WORTLEY, D. Puppilometer for use during anaesthesia. Anaesthesia, v. 39, n. 9, p. 908-10, 1984.

BATISS, B.; LYNAUGH, J. Teaching physical assessment. Nurs. Outlook, v. 23, n. 5, p. 297-302, 1975.

DE TORNYAY, R. Identifying research prionities for nursing education: Editorial. L Nurs, Educ., $v$. 26, n. 2, p. 49, 1987.

DUTTON, M.H. Assistência de acompanhamento pós-operatório em neurocirurgia. In: DRAIN, C.B.; SHIPLEY, S.B. Enfermagem na sala de recuperacäo. Rio de Janei ro, Interamericana, 1981, cap. 22 , p. 404-40.

IIA I.L, S.M.S. Ophthalmic assessment: techniques for the critical care nurse. Dimens. Crit. Care Nurs., v. 3, n. 5, p. 262-7, 1984.

KOIZ.UMI, M.S. Exame físico em pacientes agudos graves: uma experiência de ensino. Enf, Novas Dimens., v. 2, n. 5, p. 284-9, 1976.

LORD-FEROLI, K.; MAGUIRE-McGINTY, M. Toward a more onjective approch to pupil assessment. LNeurosurg. Nurs., v. 17, n. 5, p. 309-12, 1985.

MAGILL, R.A. Aprendizagem motora: conceitos e aplicaçūes. São Paulo, Edgard Bl ucher, 1984. cap. 3, p. 54-69: Sensação c Percepçāo.

MARCH, K. Look into my cyes. L. Neurosurg. Nurs., v. 15, n. 4, p. 213-21, 1983.

NORMAN, S. The pupil check. Am.J.Nurs., v. 82, n. 4, p. 588-91, 1982.

PLUM, F.; POSNER, J.B. Diagnósticode estupor e coma. $2^{2}$ ed., Rio de Janeiro, Guanabara Koogan, 1977. cap.1, p. 1-82: Fisiopatologia dos si nais e sintomas do coma ana tomi a juncional.

ROLFSEN, M.L.; DA VIS, W.R. Cerebral function and preservation during cardiac arrest. Crit. Care. Med., v. 17, n. 3, p. 283-92, 1989.

SAVOY, S.M. The craniotomy patient: identifying the patient's neurological status. AORNJ., v. 40, n. 5, p.716-24, 1984.

SIMŌES, E.A.Q.; TIEDMANN, K.B. Psicologia da percepcāo. Sāo Paulo, EPU, 1985.

SOUSA, R.M.C. Avaliaçåo de use de uma "Espátula_Pupilômeto" (E.P.). Sāo Paulo, 1989, 59 p. Dissertação (Mestrado) - Escola de Enfermagem da USP, Universidade de Sāo Paulo.

STEEN-HANSEN, J.E; et al. Pupil size and light reacti vity during cardiopulmonary resuscitation: a clinical study. Crit. Care Med., v. 16, n. 1, p. 69-70, 1988.

TRA VERS, R.M.W. Essentials of leaming. 4.ed. New York, Mac Millan, 1977. cap. 9, p. 298-346: Perceptionand percentual learning.

TRUNKEY, D.D. Trauma. Sci.Am., v. 249, n. 2, p. 20-7, 1983.

VOIGHT, J.W. Physical assessment skills in the curriculum: a pilot-project \& follow-up.J. Nurs. Educ., v. 19, n. 2, p. 26-30, 1980.

WILSON, S.F; et al. Determining interrater reliability of nurses assessment of pupillary size and reaction. L.Neuros. Nurs., v. 20, n. 3, p. 189-92, 1988. 